\title{
COMMON DRIVING NOTIFICATION PROTOCOL BASED ON CLASSIFIED DRIVING BEHAVIOR FOR COOPERATION INTELLIGENT AUTONOMOUS VEHICLE USING VEHICULAR AD-HOC NETWORK TECHNOLOGY
}

\author{
Che-Hung Lin, Fang-Yan Dong, Kaoru Hirota \\ Department of Computational Intelligence and Systems Science \\ Tokyo Institute of Technology G3-49, 4259 Nagatsuta, Midori-ku \\ Yokohama 226-8502, Japan
}

\begin{abstract}
A protocol, called common driving notification protocol (CDNP), is proposed based on the classified driving behavior for intelligent autonomous vehicles, and it defines a standard with common messages and format for vehicles. The common standard format and definitions of CDNP packet make the autonomous vehicles have a common language to exchange more detail driving decision information of various driving situations, decrease the identification time for one vehicle to identify the driving decisions of other vehicles before or after those driving decisions are performed. The simulation tools, including NS3 and SUMO, are used to simulate the wireless data packet transmission and the vehicle mobility; the experiment results present that the proposed protocol, CDNP, can increase the reaction preparing time with maximum value 250 seconds, decrease the identification time and the average travel time. Prospectively, it is decided to implement the CDNP as a protocol stack in the Linux kernel to provide the basic protocol capability for real world transmission testing.
\end{abstract}

\section{Introduction}

In [1-5], various lane detection algorithms and path-planning algorithms are proposed for vehicles to realize the autonomous driving. The driving behavior of vehicles driving on the road, however, is always affected by other vehicles. The autonomous driving in [1-5], however, is standalone. The driving decision information of other vehicles is not considered. That means autonomous vehicles did not cooperate with each other to make a more suitable driving decision. The cooperation means transfer/receive information to/from other vehicles and adjust their driving decisions. According to [6], 60 percent of accidents could be avoided if the vehicle were provided with information half a second before the moment of collision. To realize the autonomous driving in our daily life, the cooperation between vehicles is necessary [7]. To achieve cooperation, the most important thing is driving information exchange. The vehicular ad-hoc network (VANET) [8-10] provides the capability for vehicles to communicate with each other. The emergency message $[11,12]$ is proposed to provide emergency warning. However, the accident warning is just a part of the reason affecting the driving decisions of vehicles. In [13], the vehicle control algorithms for cooperative driving is proposed. The four trajectory planning algorithms [14] determine the driving plans to avoid collision at crossings using inter-vehicle communication. The cooperative driving in $[7,13,14]$ can satisfy special situations 
and usages. However, to achieve autonomous driving in various driving situations, more detail driving information and standardized common protocol for each car manufacturer are necessary. The normal driving decision information of other vehicles is the key for autonomous vehicle to make a more suitable driving decision and avoid accidents. If the emergency warning message is considered as the treatment, the normal driving information will be the prevention. There is, however, no standardized protocol to disseminate the normal driving decision information for autonomous driving to improve the driving safety and achieve cooperated autonomous driving. Therefore, the objective of this proposal is to propose a common protocol as a standard for autonomous vehicles.

A common driving notification protocol (CDNP) is proposed based on the various classified driving behaviors for autonomous vehicles. It is a protocol designed below the application layer. It focuses on not only the safety warning message dissemination, more importantly, but also normal driving information. The disseminations of normal driving information can realize the "talking" between vehicles using VANET technology. The CDNP is a common language between vehicles. It defines a standard with the common message format with a series of message types and codes, so each vehicle is able to understand what the driving decision other vehicle wants to do. It can be used to instead of the function of car light signal or horn. The human driver normally determines the driving actions of other vehicles by identifying the car light signal of other vehicles. For autonomous vehicle, however, the time of image processing $[15,16]$ is necessary to identify the driving actions of other vehicles. The CDNP enabled vehicles is termed cooperation intelligent autonomous vehicle in this proposal.

The CDNP provides more detail driving decision information of autonomous vehicles. With the CDNP, each vehicle can understand the driving situations or intentions of other vehicles without image or audio processing algorithm. With the CDNP, the autonomous vehicle can get not only the information from its sensors, but more importantly, the information about the position, speed, and driving decisions or intentions from other autonomous vehicles. The autonomous vehicles can make a more suitable driving decision according to the common messages stored in the CDNP packet header. The CDNP changes the passive detection of the driving decision of other vehicles into proactive notification to other vehicles about its driving decision. It also makes the identification of the driving decisions of other vehicles before the decisions performed possible. Therefore, the identification time (IT) is decreased. The identification time is defined as the necessary time for one vehicle identify the driving decisions of the other vehicles. Furthermore, the reaction preparing time (RPT) is increased. The reaction preparing time means the buffer time period of one vehicle to prepare a suitable reaction to react to the other vehicle's driving decision before the driving decision is performed. Furthermore, the average traveling time to the destination can also be decreased when in a hurry. The CDNP protocol can also be extended for more complex driving behaviors to make the cooperation intelligent autonomous vehicle more intelligent.

The open source simulator and tool are used in the simulation, including NS-3 [17], and simulation of urban mobility (SUMO) [18]. The NS-3 is a simulator used to simulate the network communication, and the SUMO allows to simulate the realistic movement of vehicles through a given road network. The two simulation tools are used to simulate the movement and communications of each vehicle at the same time. The reaction time and vehicle traveling time are examined.

In 2, the common driving notification protocol is proposed, including its concept, packet message format, and message definition. The concept of the cooperation intelligent autonomous driving based on the CDNP is shown in 3. The CDNP based cooperation intelligent autonomous driving simulations and results in different scenarios are described in 4. In 5, the vehicle moving characteristic based on the CDNP are discussed.

\section{Architecture and Message For- mat of Common Driving Notifi- cation Protocol (CDNP)}

The main reason causing the car accident is unsafe driving, e.g., changing driving lanes without making sure there are no cars next to itself, chang- 
ing its driving speed suddenly, and turning the driving direction without any signal. The driving decisions of vehicles are always affected by other vehicles. Therefore, the cooperative driving is necessary.

The human driver can notify other driver about his/her driving actions by flashing the car light or sounding the horn. The human driver can also determine the driving actions of other vehicles by identifying the car light signal of other vehicles or hearing the sound of the horn. For the autonomous, however, the image processing algorithm is necessary to identify the car light, and the digital audio processing algorithm is necessary to identify the horn. These algorithms may not work well if the environment is full of interference, e.g., heavy rain, noise environments. When one autonomous vehicle performs its driving decision, if it is possible to notify other autonomous vehicle about the driving decision using another support method, the autonomous driving environment will be safer.

The IEEE 802.11p WAVE standard [19] defines the technology for use in the dedicated shortrange communications (DSRC) band [20], and the IEEE 1609.4 [21] defines a multi-channel WBSS operation. Furthermore, many VANET transmission protocols are proposed, such as the adaptive connectivity aware routing (ACAR) [22], the urban vehicular broadcast (UVCAST) [23], the distribution-adaptive distance with channel quality (DADCQ) protocol for multi-hop broadcasting [24], etc. Those VANET technologies can be utilized to realize the communication between vehicles.

To achieve the cooperative driving, the most important thing is to let vehicle efficiently identify the driving decision of other vehicles. Just like as the human driver can judge the information from the car light. Hence, the CDNP is proposed to make the notification more reliable and efficient.

In Figure 1, the comparison between normal autonomous driving and autonomous driving with CDNP is shown. It is shown that a longer processing time delay is necessary for other vehicles to identify the driving decision of vehicle A using its sensors and the image processing / digital audio processing algorithm. The CDNP is designed to be immediately transferred just after the driving decision is decided. If the CDNP packet is re- ceived by other vehicles before the driving decision is performed, there will be enough processing time for the autonomous driving system to make a suitable control of the vehicle. Even though the CDNP packet is received after the driving decision of vehicle A is performed. The identification time (IT) will still shorter than the image / digital audio processing time. In the worst case, if the CDNP is received after the image / digital audio algorithm finished. The autonomous vehicle can still use the result obtained from the algorithm. Hence, the CDNP provides other reliable method to identify the driving decisions of other vehicles. This proposal focuses on the CDNP notification. The autonomous driving control, e.g., obstacle avoidance, keep a safe distance, decision conflicts avoidance, is out of range of this proposal.

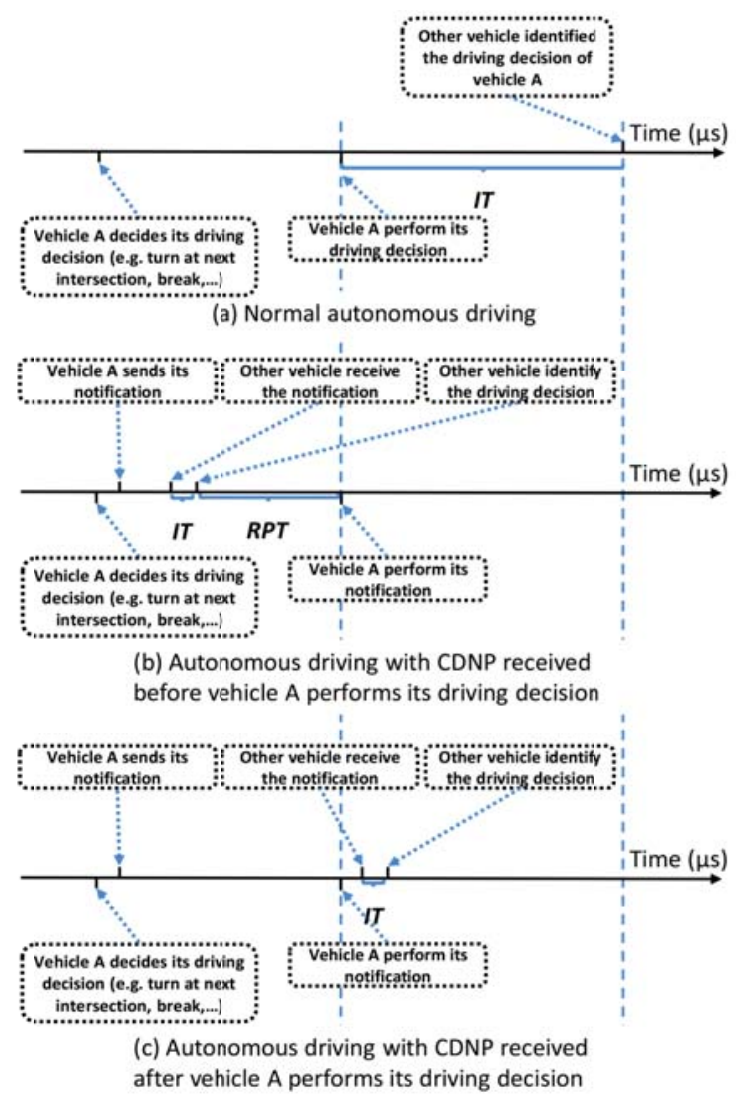

Figure 1. Comparison between normal autonomous driving and autonomous driving with the common driving notification protocol (CDNP). 


\subsection{Architecture of CDNP}

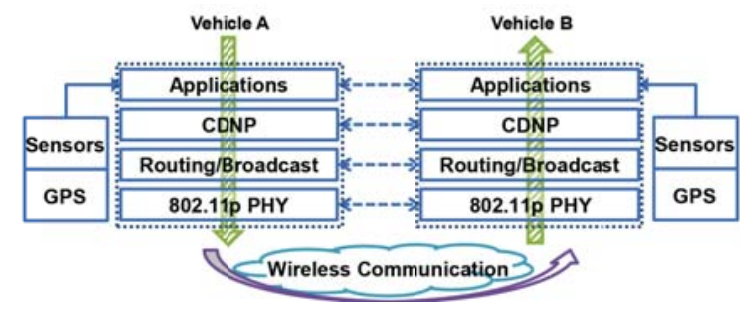

Figure 2. The architecture of the common driving notification protocol (CDNP).

The architecture of CDNP is shown in Figure 2. The CDNP is based on classified driving behavior and below the application layer. The position of CDNP is designed to compatible with the facilities layer of ITS station architecture standardized in ISO/ETSI [25]. The function of CDNP is to provide the application support and information support. Therefore, it is easy to integrate the CDNP into the architecture of ITS. It plays an important role to make the vehicle understand what the other vehicles notify. The CDNP is designed to exchange common messages between vehicles using the VANET technology, including routing protocols, broadcast protocols, or unicast. The VANET beacon mechanism, i.e., basic safety message (BSM) [20, 26-28], is enabled so that each vehicle can know the related geographic position, velocity and direction of neighboring vehicles. The beacon messages are periodically exchanged with neighboring vehicles in a broadcast function. The information contained in the beacon message can be temporarily stored in the memory space of the vehicle received the message. The related position information is used for the vehicle to decide the destination of the CDNP packet.

When one vehicle wants to perform some driving decisions, such as changing the driving lane, driving direction, speed, etc., the autonomous driving system installed in the application layer of its vehicle telematics system will create the CDNP message, including the type and code, according to the corresponding driving decision. The vehicles that send the CDNP packets are called source vehicles (SV), and the vehicles received the CDNP packets are called the current vehicles $(\mathrm{CV})$ in this proposal. Under the CDNP layer, some protocols can be used to ensure the data reliability of VANET wireless transmission, e.g., [29]. Normally, the CDNP is used to notify the other vehicles near to it- self using unicast or broadcast. It, however, can also be transferred to remote vehicles for special usage, e.g., traffic management of specific roads by urban traffic management system. The urban traffic management system can use the CDNP to manage the traffic flow of the urban area; in this case, the CDNP packets will be transferred to specified vehicles on specified road segment using VANET routing protocols, and the location service [30] is used to determine the destination. The traffic management is not discussed in this proposal.

\subsection{Header Format of CDNP}

To make the vehicles understand what another vehicle notifies, a specific common message format is necessary. Therefore, the format of the header fields of CDNP packets is defined and shown as Table 1. The different types and codes are simultaneously used to identify different driving notifications in CDNP. The value of type field indicates the different main categories of driving decisions. Each type includes its corresponding codes to indicate which driving decision the vehicle wants to notify. The same with many Internet protocols, the checksum here is also used for error checking and verifying the correctness of packets. The value of the checksum is calculated from the type field of the CDNP packet to the end of optional data. The length of optional data field is varied according to the value stored in type and code. This makes the CDNP flexible and extendable.

Table 1. Formats of header fields of CDNP packet.

\begin{tabular}{|l|l|l|}
\hline 0 & \multicolumn{1}{|c|}{16} & 31 \\
\hline Type & Code & Checksum \\
\hline Optional Data \\
\hline
\end{tabular}

\subsection{Types and Codes of CDNP Header}

In Table 2, all types and codes defined in the CDNP are listed, including its types, type names, descriptions, codes, and code names. There are total 9 types. They are defined based on classified driving behavior. They can be classified into four main categories: special type (type 0x00), different driving behavior (from type 0x01 to type 0x05), vehicle emergence warning information (type 0x06 and $0 x 07$ ), and urban traffic management (type 0xFF). For each type, different corresponding codes 
are defined according to different driving decisions, descripting in the following. The CDNP packet contained type $n$ and code $m$ is denoted by $\operatorname{CDNP}(n, m)$ packet and called type $n$ code $m$ CDNP packet.

The type $0 \mathrm{x} 00$ is a special type and is reserved for special types of emergency vehicles, e.g., police car, ambulance, fire engine, in emergency situations.

The special type vehicles have the highest priority to go through the road in the emergency situations, such as the police car pursues an escaped prisoner or ambulance transports the injured. When an emergency situation occurs, the special vehicles can set the type to $0 \mathrm{x} 00$ and insert necessary information into optional data. The format of optional data is different according to the different types, described in the following subsection. The type $0 \mathrm{x} 00$ must be associated with type $0 \mathrm{x} 01,0 \mathrm{x} 02,0 \mathrm{x} 03$, or 0x04, and the code field is used to indicate which type is associated. The corresponding codes for the associated type is also stored in the optional data field.

\subsection{Optional Data Format of the CDNP Header}

The optional data field is varied according to the type value stored in the CDNP header. The common formats of optional data field for each type are defined for correctly encapsulating and de-capsulate the packet header. The formats of optional data fields of the CDNP header are shown from Table 3 to Table 6 .

Optional Data Format

Table 3. Formats of optional data fields of type 0x00 and 0xFF CDNP packet.

\begin{tabular}{|l|l|l|}
\hline $\mathbf{0} \quad \mathbf{8}$ & $\mathbf{1 6}$ & $\mathbf{3 1}$ \\
\hline Type & Code & Checksum \\
\hline $\begin{array}{l}\text { Sequence } \\
\text { ber }\end{array}$ & $\begin{array}{l}\text { num- } \\
\text { sociated CDNP }\end{array}$ \\
\hline \multicolumn{2}{|l|}{ Notification Timestamp } \\
\hline
\end{tabular}

The Table 3 shows the optional data definition for type 0x00 and 0xFF. Because the type 0x00 and OxFF CDNP header should associate with other type and code, the optional data field includes the information about the associated type and the sequence number of associated CDNP packet. Those fields are used by the vehicle which received the notification to match the associated CDNP packet. The notification timestamp field stores the timestamp when the source node transfers the CDNP packet.

When the $\mathrm{CV}$ received the type $0 \mathrm{x} 00 \mathrm{CDNP}$ packet and associated CDNP packet, it will know that the special vehicle is performing special work. The associated CDNP packet will be considered as a request. It is like the human driver hear the sirens of police car. If the received CDNP is type 0xFF, the $\mathrm{CV}$ will know the message is from the urban traffic control system, and the autonomous driving system will follow the instruction.

The Table 4 shows the optional data definition for type 0x01, 0x02, 0x03, and 0x04. If this CDNP packet is associated with type $0 \mathrm{x} 00$ or $0 \mathrm{xFF}$ CDNP packet, the sequence number will be filled using the same number stored in the "sequence number of associated CDNP" field of previous 0x00 or 0xFF CDNP packet header. Otherwise, a new sequence number will be generated. The speed-X and speed$\mathrm{Y}$ field stores the speed of the vehicle that sent the notification. When the source node wants to transfer the CDNP packet, the timestamp transferring the CDNP packet is stored in the notification timestamp field. The execution timestamp stored the timestamp that the driving decision may be performed if it is calculable. Otherwise, 0 will be stored. For example, if the one autonomous vehicle wants to change its driving direction at the next intersection, it is easy for the autonomous driving system to predict the necessary time period to drive to the intersection. It is useful to warn the vehicles which are near to the intersection. If the execution timestamp is larger than 0 , the SV will re-transmit the same notification 1 packet/second to ensure the other vehicles surely receive the notification. This is because the vehicles are always moving. The CV received the notification, which execution timestamp is larger than 0 , can also predict the relative position to judge whether it is necessary to pay attention to the driving decision. If it is not necessary, the $\mathrm{CV}$ can just drop the CDNP packet. 
Table 2. Types and codes of CDNP packet.

\begin{tabular}{|c|c|c|c|c|}
\hline Type & Type meaning & Description & Code & Code meaning \\
\hline 0x00 & Special type & $\begin{array}{l}\text { Reserved for special types of vehicles, } \\
\text { such aspolice car, ambulance, fire } \\
\text { engine, etc. It is only set for emergency } \\
\text { situations, e.g., pursuing an escaped } \\
\text { prisoner, and cannot be used by normal } \\
\text { vehicles. }\end{array}$ & $\begin{array}{l}0 \times 00 \\
0 \times 01 \\
0 \times 02 \\
0 \times 03\end{array}$ & $\begin{array}{l}\text { Associate with type } \\
\text { 0x01 } \\
\text { Associate with type } \\
\text { 0x02 } \\
\text { Associate with type } \\
\text { 0x03 } \\
\text { Associate with type } \\
\text { 0x04 }\end{array}$ \\
\hline 0x01 & Changing lanes & $\begin{array}{l}\text { Used when one vehicle wants to change } \\
\text { its driving lanes and may affect the } \\
\text { other vehicles. }\end{array}$ & $\begin{array}{l}0 \mathrm{x} 00 \\
0 \mathrm{x} 01\end{array}$ & $\begin{array}{l}\text { Left } \\
\text { Right }\end{array}$ \\
\hline 0x02 & Changing speed & $\begin{array}{l}\text { Used when one vehicle wants to } \\
\text { dramatically change its driving speed } \\
\text { for special purposes and may affect the } \\
\text { other vehicles. }\end{array}$ & $\begin{array}{l}0 \times 00 \\
0 \times 01 \\
0 \times 02 \\
0 x 03\end{array}$ & $\begin{array}{l}\text { Speedup } \\
\text { Speed-down } \\
\text { Emergency brake } \\
\text { Back a car }\end{array}$ \\
\hline $0 \times 03$ & $\begin{array}{l}\text { Changing } \\
\text { direction }\end{array}$ & $\begin{array}{l}\text { Used when one vehicle want to change } \\
\text { its driving direction and may affect the } \\
\text { other vehicles. } \\
\text { Normally used before the intersections, } \\
\text { and it canalso be used in the situation of } \\
\text { turning the direction to parking spaces. }\end{array}$ & $0 \times 01$ & Right \\
\hline 0x04 & Overtake & $\begin{array}{l}\text { Used when one vehicle wants to } \\
\text { overtake other vehicles. }\end{array}$ & $0 \times 00$ & Overtake notification \\
\hline 0x05 & $\begin{array}{l}\text { Unsafe reply to } \\
\text { the notification }\end{array}$ & $\begin{array}{l}\text { Used to warn another vehicle about the } \\
\text { driving decision. }\end{array}$ & $0 \times 00$ & Unsafe \\
\hline 0x06 & $\begin{array}{l}\text { Breakdown } \\
\text { notification }\end{array}$ & Used when one vehicle breakdown. & $\begin{array}{l}0 \times 00 \\
\text { 0x01 } \\
\text { 0x02 }\end{array}$ & $\begin{array}{l}\text { Minor failure } \\
\text { Medium failure } \\
\text { Hard failure }\end{array}$ \\
\hline 0x07 & $\begin{array}{l}\text { Environment } \\
\text { emergency } \\
\text { warning }\end{array}$ & $\begin{array}{l}\text { Used to alert other vehicles about the } \\
\text { environmental emergency. }\end{array}$ & $\begin{array}{l}0 \times 01 \\
0 \times 02\end{array}$ & $\begin{array}{l}\text { Minor emergency } \\
\text { warning } \\
\text { Medium emergency } \\
\text { warning } \\
\text { Hardemergency } \\
\text { warning }\end{array}$ \\
\hline $0 \mathrm{xFF}$ & Global command & $\begin{array}{l}\text { Reserved for global traffic flow control. } \\
\text { It can only be used by the urban traffic } \\
\text { management systemin special situations, } \\
\text { such as decrease the traffic flow of } \\
\text { specified road segments or block } \\
\text { specified road segments in some } \\
\text { emergency reasons. }\end{array}$ & 0x02 & $\begin{array}{l}\text { Associate with type } \\
\text { 0x01 } \\
\text { Associate with type } \\
\text { 0x02 } \\
\text { Associate with type } \\
\text { 0x03 }\end{array}$ \\
\hline
\end{tabular}


Table 4. Formats of optional data fields of type 0x01, 0x02, 0x03, and 0x04 CDNP packet.

\begin{tabular}{|c|c|c|}
\hline 8 & 16 & 31 \\
\hline Type & Code & Checksum \\
\hline $\begin{array}{l}\text { Sequence } \\
\text { ber }\end{array}$ & num- & Unused \\
\hline Speed-X & & Speed-Y \\
\hline \multicolumn{3}{|c|}{ Notification Timestamp } \\
\hline \multicolumn{3}{|c|}{ Execution Timestamp } \\
\hline
\end{tabular}

Table 5. Formats of optional data fields of type 0x05 CDNP packet.

\begin{tabular}{|l|l|l|}
\hline $\mathbf{0} \quad \mathbf{8}$ & $\mathbf{1 6}$ & $\mathbf{3 1}$ \\
\hline Type & Code & Checksum \\
\hline $\begin{array}{l}\text { Sequence } \\
\text { ber }\end{array}$ & num- & $\begin{array}{l}\text { Unsafe CDNP Sequence } \\
\text { Number }\end{array}$ \\
\hline \multicolumn{3}{|r|}{ Reply Timestamp } \\
\hline
\end{tabular}

The Table 5 shows the optional data definition for type 0x05 and 0x06. The "unsafe CDNP sequence number" field is the same with the sequence number stored in the notification packet that the CV wants to notify to. Its' function is like the human driver honk the horn to warn other drivers.

Table 6. Formats of optional data fields of type 0x06 and 0x07 CDNP packet.

\begin{tabular}{|l|l|l|}
\hline $\mathbf{0} \quad \mathbf{8}$ & $\mathbf{1 6}$ & $\mathbf{3 1}$ \\
\hline Type & Code & Checksum \\
\hline Identifier & & Emergency Information \\
\hline \multicolumn{3}{|c|}{ Notification Timestamp } \\
\hline
\end{tabular}

Table 6 shows the optional data definition for type 0x06 and type 0x07. For type 0x06, the identifier indicates the failure parts of the $\mathrm{CV}$, and the parts of the vehicle are not discussed in this proposal; the emergency information stores the information for the manufacturer. For type 0x07, the identifier indicates the emergency situations that the vehicle detected using its sensors, including accidents, slippery road surfaces, road surface pit, chatter bump, static obstacle, moving obstacle, animal, etc. The emergency information field stores various emergency situations information.

\section{Concept of Cooperation Intelli- gent Autonomous Driving based on CDNP}

In this section, the concept application of CDNP, cooperation intelligent autonomous driving based on CDNP, is introduced. All autonomous vehicles can understand what the other vehicles notified according to the standard message format, types, and codes defined in CDNP.

It is assumed that autonomous driving technologies are already embedded in each vehicle. It also is assumed that each autonomous vehicle equips with global positioning system (GPS) device and numerous sensors with such techniques as radar, lidar, and computer vision to sense their surrounding environment for safety autonomous driving. In this proposal, only the vehicle driving decision information transmission is discussed; the autonomous driving technologies, such as however to control the vehicle, lane detection, and obstacle avoidance, etc., are out of the range of this proposal, so they are not discussed in this proposal. The VANET wireless interface is also installed. The VANET wireless interface is used to transmit or receive the data packets from other vehicles or infrastructures. The vehicle driving control application that is the application layer user interface is also available for passenger to input his driving requirements, such as the destination, in a hurry or not, etc.

\subsection{Sending Procedure of the Cooperation Intelligent Autonomous Driving}

The flow of sending procedure of the cooperation intelligent autonomous driving based on the CDNP is shown as Figure 3. 


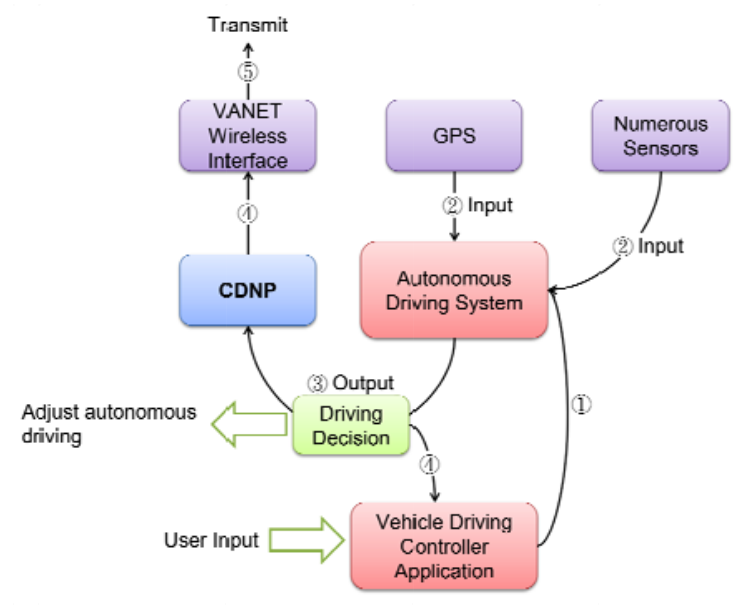

Figure 3. Sending procedure of the cooperation intelligent autonomous driving with the common driving notification protocol (CDNP).

When the vehicle received driving request inputted by passengers using the vehicle driving control application, the driving command information will be forwarded to the autonomous driving system. Then, the system will decide a most suitable driving decision according to the information, gathered from the GPS and various sensors to ensure the driving safety. The driving decision is used to adjust the autonomous driving of the vehicle. At the same time, the CDNP packet is generated. The CDNP packet is transmitted using the VANET wireless communication to notify other vehicles about the driving decision. It is like the human driver can notify other drivers using car light. Because the geographic positions of neighboring vehicles are available via the beacon mechanism, the CDNP can be transmitted to specific vehicles using unicast. Broadcast can also be used to notify a group of vehicles, e.g., all vehicles near to the intersection. In the case of broadcast, the CDNP will not be re-broadcast by other vehicles. Hence, there is no broadcast storming problem. To ensure the reliable CDNP packet transmission, the TCP liked protocol, such as TFRC-FC-SACK [31], which has better performance in VANET environment, can be used in the transport layer.

\subsection{Receiving Procedure of the Coopera- tion Intelligent Autonomous Driving}

The flow of receiving procedure of the cooperation intelligent autonomous driving based on the CDNP is shown as Figure 4. When the CV re- ceived the packets transferred from other vehicles, it will de-capsulate the packet and check whether the packet is a CDNP packet.

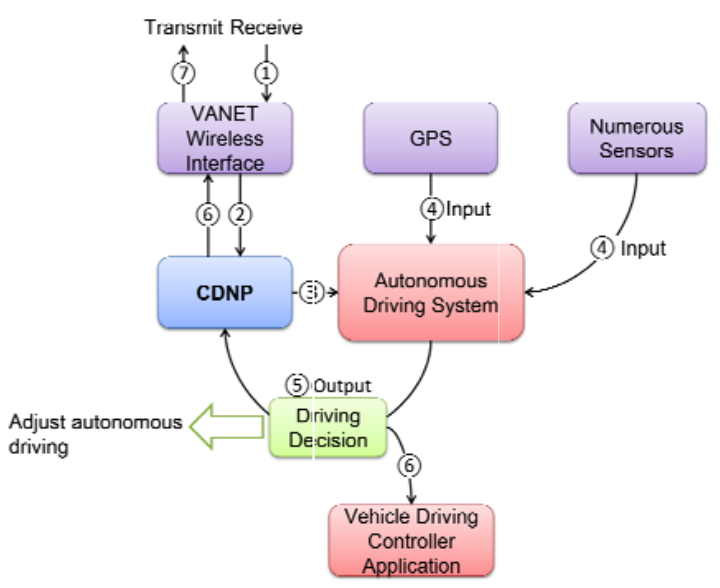

Figure 4. Receiving procedure of the cooperation intelligent autonomous driving with the common driving notification protocol (CDNP).

If it is, the CDNP packet will be forwarded into the autonomous driving system. Then the system can judge whether it is necessary to perform some reaction or not. If it is necessary, the system can decide a suitable driving decision according to the type and code stored in the CDNP header and the information gathered from the GPS and various sensors. After the driving decision is decided, the decision will be used to adjust the autonomous driving of the vehicle and display some information for the passengers about the driving decision if necessary. Of course, if the autonomous driving system detects the possibility of unsafe driving decision, it can also transmit unsafe reply CDNP to the vehicle which sends the notification. It is like the human driver honk the horn to warn other drivers.

\subsection{CDNP based Autonomous Driving Compared with Normal Autonomous Driving and Human Drivers}

Table 7 shows the comparison of CDNP based autonomous driving, normal autonomous driving and human driver. The normal autonomous driving means the autonomous driving just depends on the sensors and various algorithms to control the vehicle. 
Table 7. Comparison of different driving decision identification.

\begin{tabular}{|c|c|c|}
\hline $\begin{array}{l}\text { CDNP based } \\
\text { Autonomous } \\
\text { Driving }\end{array}$ & $\begin{array}{l}\text { Normal Au- } \\
\text { tonomous } \\
\text { Driving }\end{array}$ & $\begin{array}{l}\text { Human } \\
\text { Driver }\end{array}$ \\
\hline Not tired & Not tired & $\begin{array}{l}\text { Long- } \\
\text { distance } \\
\text { driving will } \\
\text { be tired }\end{array}$ \\
\hline $\begin{array}{l}\text { No processing } \\
\text { time }\end{array}$ & $\begin{array}{l}\text { Longer time } \\
\text { for im- } \\
\text { age/audio } \\
\text { processing }\end{array}$ & $\begin{array}{l}\text { No process- } \\
\text { ing time }\end{array}$ \\
\hline $\begin{array}{l}\text { Data transmis- } \\
\text { sion time is } \\
\text { necessary }\end{array}$ & $\begin{array}{l}\text { No data trans- } \\
\text { mission time }\end{array}$ & $\begin{array}{l}\text { No data } \\
\text { transmission } \\
\text { time }\end{array}$ \\
\hline $\begin{array}{l}\text { Easy to locate } \\
\text { driving lane of } \\
\text { the source far } \\
\text { behind it }\end{array}$ & Not easy & $\begin{array}{l}\text { It's a danger- } \\
\text { ous driving }\end{array}$ \\
\hline $\begin{array}{l}\text { No sensor nec- } \\
\text { essary }\end{array}$ & $\begin{array}{lr}\text { Various } & \text { sen- } \\
\text { sors } & \text { are } \\
\text { necessary } & \end{array}$ & $\begin{array}{l}\text { No sensor } \\
\text { necessary }\end{array}$ \\
\hline $\begin{array}{l}\text { Operable } \\
\text { range of ap- } \\
\text { proximately } \\
250 \mathrm{~m}\end{array}$ & $\begin{array}{l}\text { Long distance } \\
\text { may not work } \\
\text { properly due } \\
\text { to the image } \\
\text { resolution }\end{array}$ & $\begin{array}{l}\text { Depend on } \\
\text { the vision }\end{array}$ \\
\hline
\end{tabular}

According to the Table 7, it is known that the CDNP notification can provide human liked driving decision identification than normal autonomous driving technologies. Of course, it is better to integrate the CDNP and normal autonomous driving technologies at the same time to provide a safer driving environment for passengers.

\subsection{Transfer Sequence Different between Type 0x00 (or 0xFF) CDNP and Other Types}

The type $0 \mathrm{x} 00$ and type $0 \mathrm{xFF}$ are special types designed for special usages. The type 0x00 is used to increase the notification priority. It makes other vehicles know that the source vehicle is a special vehicle and it is performing a special work. The CDNP packet associated by the type 0x00 will be regarded as a request. The Figure 5 shows an example scenario of type $0 \times 00$ notification. In this example, the vehicle $\mathrm{A}$ is a special vehicle, such as police car, and it is performing emergency work. Therefore, it transmits the type 0x00 CDNP packet to associate its driving lane change notification. The vehicle received the notification will consider the notification as a high priority request. It is like the police car uses its siren to request other vehicles to make way in emergency situations.

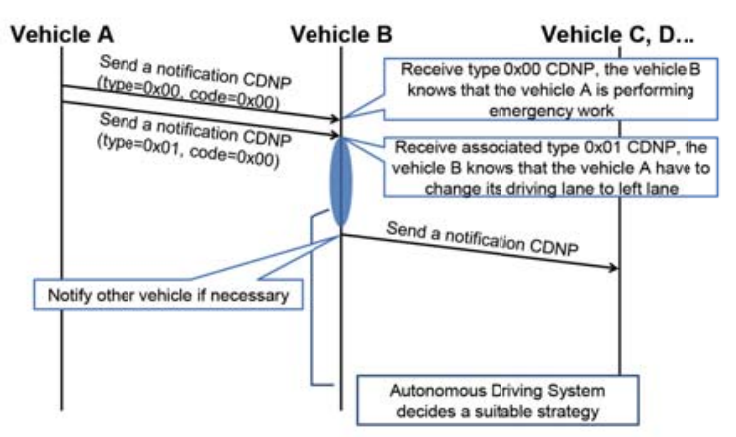

Figure 5. Example scenario of type 0x00 (associate with type 0x01).

The type $0 x F F$ is reserved for the urban traffic management system to perform the traffic management. It changes the notification into the command. The associated CDNP packet is regarded as a command. All of the vehicles received the type 0xFF CDNP will follow the instruction from the urban traffic management system. This is useful if specific road segments must be blocked in special emergency situation. The Figure 6 shows an example of type OxFF notification.

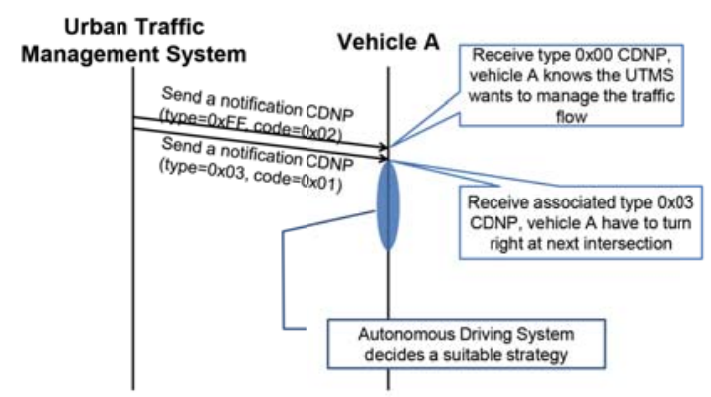

Figure 6. Example scenario of type 0xFF (associate with type 0x03).

\section{CDNP Simulations and results}

The simulations and performance analyses are conducted to verify the efficiency of the proposed common driving notification protocol in this section. Because there still are no autonomous ve- 
hicles driven in our daily life, the simulators are used to simulate the cooperated driving concept of the autonomous vehicles. In the simulations, each autonomous vehicle equipped with OBUs that contains VANET wireless communication interface with a transmission range of $250 \mathrm{~m}$, so that autonomous vehicles can communicate with neighboring vehicles. The VANET wireless link can only be established if the distance between two nodes is less than their transmission range, and the beacon mechanism is enabled. The simulation environment, simulation scenarios, and metrics are introduced in the following.

\subsection{Simulation Environment}

The open source simulation tools, including NS-3 [17] and simulation of urban mobility (SUMO) [18], are used to simulate the wireless data transmission and vehicle movement. The programming language used is $\mathrm{C}++$.

NS-3: the NS-3 is a discrete-event network simulator for building Internet systems, including wired and wireless communication. It is used to simulate the wireless data transmission of VANET.

SUMO: the SUMO allows to simulate the realistic vehicle movement, which consists of vehicles moves through a given road network. Numerous of the car following model are implemented in SUMO for more reality vehicle movement simulation.

The SUMO can be connected with other applications through a TCP-based client-server architecture using Traffic Control Interface (TraCI) [32]. The TraCI in SUMO allows other application to retrieve values of simulated objects, e.g., vehicles, traffic lights, lane status, and to manipulate their behavior, e.g., change driving lane, change driving speed, change driving route, change traffic light, and stop the vehicle. Therefore, through the TraCI, the NS-3 can achieve online interaction with SUMO.

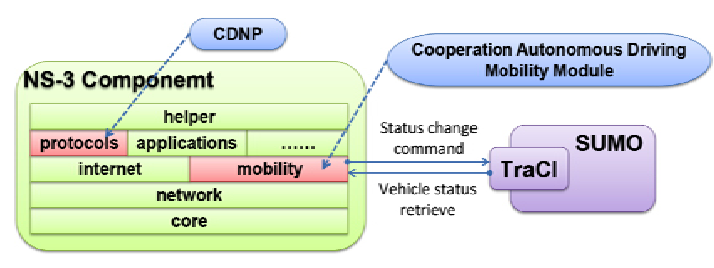

Figure 7. Implementation of CDNP and cooperation autonomous driving mobility model.

The CDNP and simple basic capability of autonomous driving are implemented as a sub-module of NS3. The component implementations are shown as Figure 7.

Table 8. List of important simulation parameters setting.

\begin{tabular}{|c|c|}
\hline Simulation Time & $400 \mathrm{sec}$. \\
\hline Topology Size & $\begin{array}{l}\text { Depend on the scenario de- } \\
\text { scribed in the next subsec- } \\
\text { tion }\end{array}$ \\
\hline $\begin{array}{l}\text { Number of Vehi- } \\
\text { cles }\end{array}$ & $\begin{array}{l}\text { Depend on the scenario de- } \\
\text { scribed in the next subsec- } \\
\text { tion }\end{array}$ \\
\hline $\begin{array}{l}\text { Destination } \\
\text { Selection }\end{array}$ & $\begin{array}{l}\text { According to the received } \\
\text { beacon information stored } \\
\text { in the vehicle memory }\end{array}$ \\
\hline $\begin{array}{ll}\text { Media } & \text { Access } \\
\text { Control } & \end{array}$ & $802.11 \mathrm{p}$ \\
\hline $\begin{array}{l}\text { Transmission } \\
\text { Range }\end{array}$ & $250 \mathrm{~m}$ \\
\hline $\begin{array}{l}\text { Propagation } \\
\text { Models }\end{array}$ & $\begin{array}{l}\text { TwoRayGroundPropagation } \\
\text { LossModel }\end{array}$ \\
\hline $\begin{array}{l}\text { Data } \\
\text { Type }\end{array}$ & CDNP Packet \\
\hline Data Packet Size & $\begin{array}{l}\text { Changed according to dif- } \\
\text { ferent CDNP type. }\end{array}$ \\
\hline $\begin{array}{l}\text { Beacon Message } \\
\text { Interval }\end{array}$ & 1 pkt. / sec. \\
\hline $\begin{array}{l}\text { Beacon Infor- } \\
\text { mation Stored } \\
\text { Threshold }\end{array}$ & $30 \mathrm{sec}$. \\
\hline $\begin{array}{ll}\text { Vehicles } & \text { Move- } \\
\text { ment } & \end{array}$ & $\begin{array}{l}\text { Randomly generated by } \\
\text { SUMO }\end{array}$ \\
\hline $\begin{array}{l}\text { Traffic Light In- } \\
\text { terval }\end{array}$ & $\begin{array}{l}\text { Randomly generated by } \\
\text { SUMO }\end{array}$ \\
\hline
\end{tabular}

CDNP: the CDNP is implemented as a protocol component in NS-3. 
Cooperation Autonomous Driving Mobility Module: it is implemented as a mobility module to online interaction with the SUMO. It transfers control command to SUMO to handle all vehicles' driving decisions and retrieve the new vehicle statue from SUMO through the TraCI. It calculates the relative position of each vehicle to adjust their driving status, including change driving speed, change driving lane, change driving route, and stop, etc. After transferring the command to adjust the vehicle status, it also retrieves the new vehicle status from SUMO.

The important parameters and configurations are listed in the Table 8.

\subsection{Maps and Scenarios}

\section{Maps}

The highway scenario is shown as Figure 8. The highway is four-lane two-way. The length of road is $4 \mathrm{~km}$. The map of intersection scenario is shown in the Figure 9. The Figure 10 shows the digital map used for the urban scenario, and it is obtained from San Francisco city of the openstreetmap [33].

\section{Scenarios}

\section{- Wireless Transmission Simulation}

In this simulation, the intersection scenario, highway scenario, and urban scenario are used. The average number of vehicles is varied from 10 to 20 and the average speeds of each vehicle are randomly assigned between $0 \mathrm{~km} / \mathrm{h}$ and 50 $\mathrm{km} / \mathrm{h}$ in the intersection scenario. In the highway scenario, the average number of vehicles is varied from 40 to 80 and the average speeds of each vehicle are randomly assigned between $80 \mathrm{~km} / \mathrm{h}$ and $110 \mathrm{~km} / \mathrm{h}$. In the urban scenario, the start point and end point of all vehicles are randomly selected. The average number of vehicles varies from 200 to 400, and the speeds of each vehicle are randomly varied between $50 \mathrm{~km} / \mathrm{h}$ and $80 \mathrm{~km} / \mathrm{h}$. The movements of autonomous vehicles are statically generated by using SUMO. The CDNP packets are transmitted by analyzing the movement script that generated by SUMO, e.g., the vehicle change driving direction, stop.

\section{- Reaction Preparing Time Simulation}

The intersection scenario and highway scenario are used. In intersection scenario, the vehicle
1 will turn left when it arrives the intersection. The navigation information can make the autonomous driving system know it has to turn left before it arrive the intersection. The speed of the vehicle 2 is larger than vehicle 3 , and the vehicle 2 wants to overtake the vehicle 3 . The vehicle 5 is slow-down because the vehicle 7 is turning its driving direction. Hence, the vehicle 5 has to notify the vehicle 1 about the slow-down information. This intersection scenario is generated as a static movement script for NS-3. In the highway scenario, the number of vehicles is set to 60 and the average speeds of each vehicle are randomly assigned between $80 \mathrm{~km} / \mathrm{h}$ and $110 \mathrm{~km} / \mathrm{h}$.

\section{- Identification Time Simulation}

The highway scenario is used in the identification simulation. The scenario setting is the same with the highway scenario of wireless transmission simulation. In order to compare with the image processing based driving, the vehicle image in difference is also used.

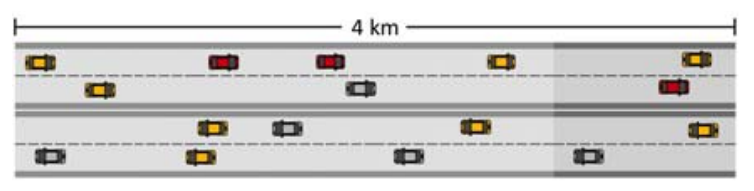

Figure 8. Highway with four-lane two-way.

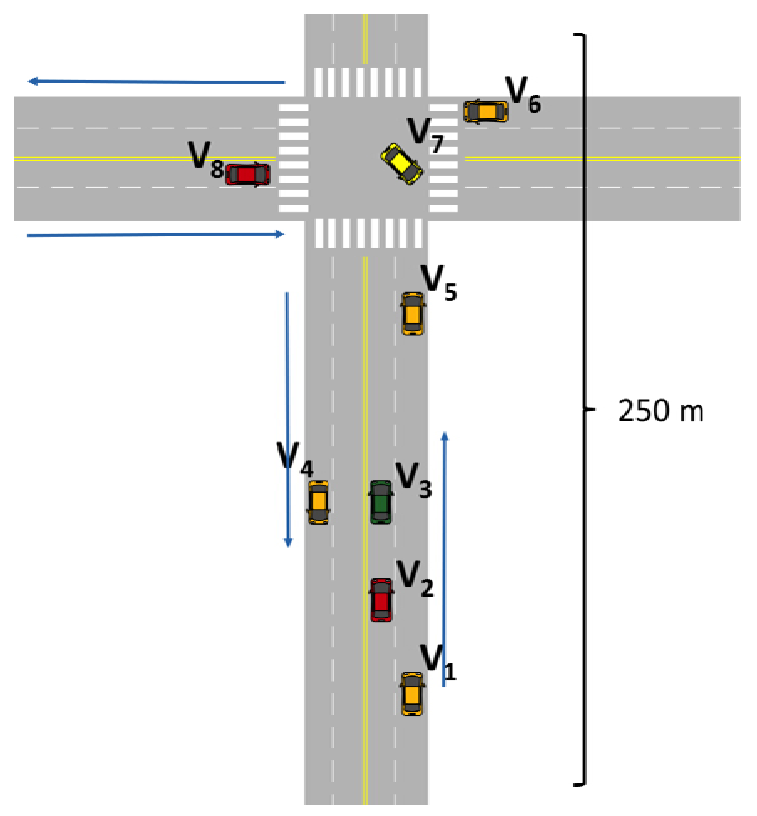

Figure 9. Intersection scenario. 


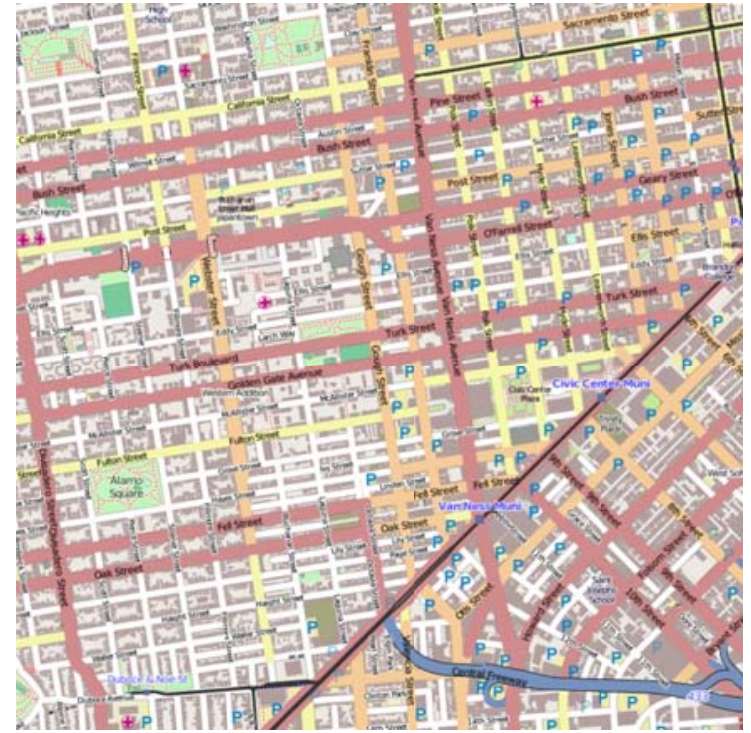

Figure 10. Digital map of San Francisco city used for the simulation of urban scenario.

- Vehicle Traveling Simulation

Two scenarios, including highway scenario and urban scenario, are used in vehicle traveling simulation. The NS-3 is configured to online interaction with the SUMO to change the vehicle moving status, e.g., driving lane, direction, speed, through the TraCI. In the highway scenario, the average number of vehicles is varied from 40 to 80 and the average speeds of each vehicle are randomly varied between $80 \mathrm{~km} / \mathrm{h}$ and $110 \mathrm{~km} / \mathrm{h}$. In the urban scenario, the start point and end point of the vehicle is randomly selected. The average number of vehicles varies from 200 to 400 , and the speeds of each vehicle are randomly varied between $50 \mathrm{~km} / \mathrm{h}$ and 80 $\mathrm{km} / \mathrm{h}$. The movements of autonomous vehicles are dynamically controlled by cooperation autonomous driving mobility module, TraCI, and SUMO.

\subsection{Simulations and Results}

- Wireless Transmission Simulation Results

In this simulation, the wireless transmission delay is compared. The result is shown as Figure 11. It shows that the end-to-end delay is varied within a certain range. This is because the CDNP is normally used to notify other vehicles in a certain range of the current vehicle when the driving decision may affect them. Therefore, the multi-hop transmission is usually not necessary. The end-to-end delay is short. In Figure 12, the transmission overhead of CDNP is shown. It is known that the CDNP will not cause large network overhead. The overhead of highway scenario is smaller than the intersection scenario. This is because the movement behavior of vehicles on highway is simpler than the movement near to the intersection.

\section{- Reaction Preparing Time Simulation Results}

According to the intersection scenario described in section 4.2, some autonomous vehicles have to transmit the CDNP notification. For example, the vehicle 2 will transfer overtake notification, the vehicle 5 will transmit slow-down notification, and the vehicle 1 will transmit turn left notification. The destination of each notification can be easily found from the stored beacon information. It can also predict the position if the driving decision is not executed immediately.

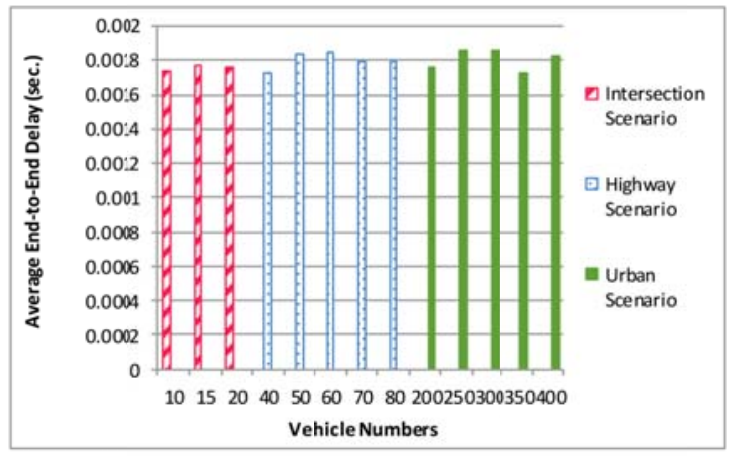

Figure 11. End-to-End delay of CDNP wireless transmission.

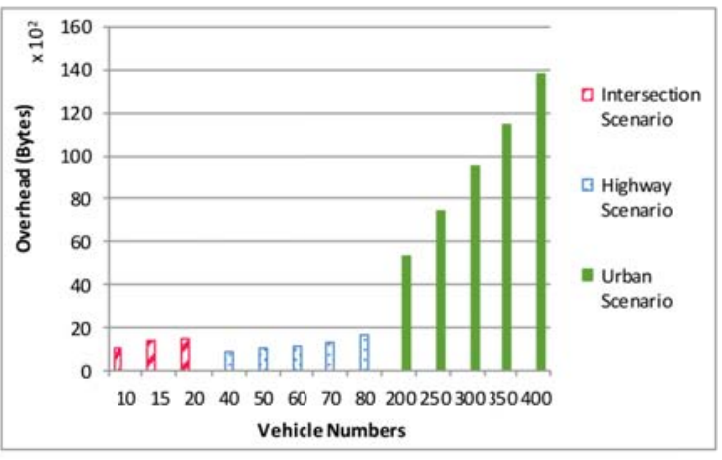

Figure 12. Overhead of CDNP wireless transmission. 
The Figure 13 shows the RPT relations between vehicles. The Vab means the reaction preparing time for vehicle a to react to vehicle $b$ before the driving decision of vehicle $b$ is performed. The number is the RPT of vehicle b. The 0 means there is no reaction preparing time. After analyzing the simulation logs, it is known that the vehicle 1 transmits the notification before it arrives the intersection. The vehicle 5 transmits the notification and performs the slow-down driving decision at the same time. Hence, it is known that the PRT will depend on the usage of notification. It will also depend on the relative speed between two vehicles.

To examine the relationship between PRT and the relative speed between two vehicles, the highway scenario is used. Each vehicle calculates the relative position when receiving the beacon message. The faster vehicle will transmit the overtake notification to the front vehicle that is slower than it. In the real world, the slower vehicle normally changes its driving lane to slow lane to let the faster vehicle passing when the driver notices the flashing car light or hear the horn. Hence, the PRT here is obtained by subtracting the time stamp that faster vehicle sent notification from the time stamp of faster vehicle close to the slower front vehicle's one meter range.

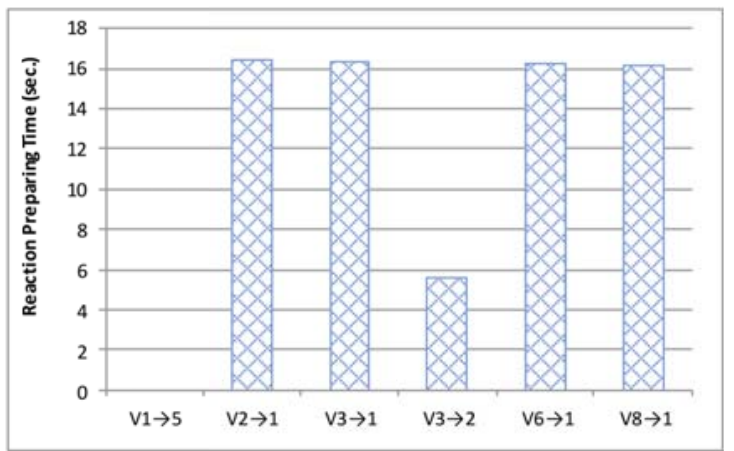

Figure 13. Reaction preparing time of intersection scenario.

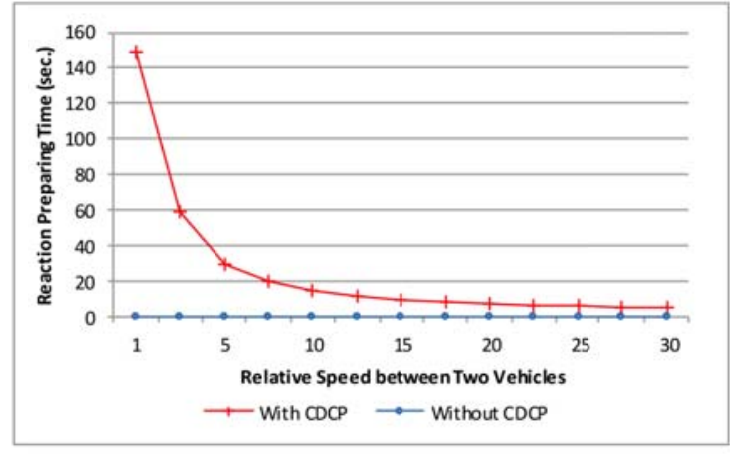

Figure 14. Reaction preparing time comparison in different relative speed between two vehicles.

The comparison of reaction preparing time when varying the relative speed between two vehicles is shown in Figure 14. It is shown that if the CDNP is available and the relative speed between two vehicles are close, the RPT will be long. When increasing the relative speed between two vehicles, the RPT is decreased. The RPT, however, may still long enough for the autonomous driving system to avoid possible accident. However, if the CDNP is not available, the driving decision cannot be transmitted. The autonomous driving system can just detect the driving decisions of other vehicles when some driving decisions have already performed. Therefore, the RPT without CDNP is 0 .

In Figure 15, it shows the relationship between the RPT and the distance when transmit the CDNP. Although the PRT is decreased if the distance between two vehicles shorter. The CDNP still increases the RPT. If the CDNP is not available, the RPT is 0 , and the autonomous driving system must react to all of the driving decisions of other vehicles after it detects the flashing car light or horn. From the results, it is known that the CDNP can increase the reaction preparing time with maximum value about $250 \mathrm{sec}-$ onds if the transmission range of VANET is $250 \mathrm{~m}$ and the distance between two vehicles is $250 \mathrm{~m}$. 


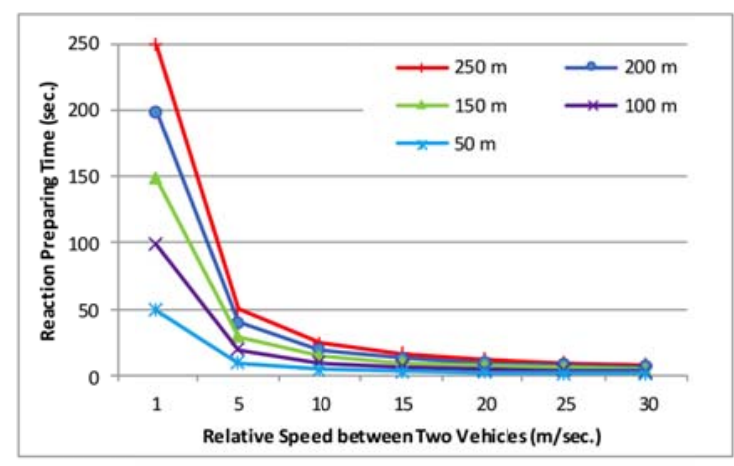

Figure 15. Reaction preparing time comparison in different distances when the CDNP is transmitted.

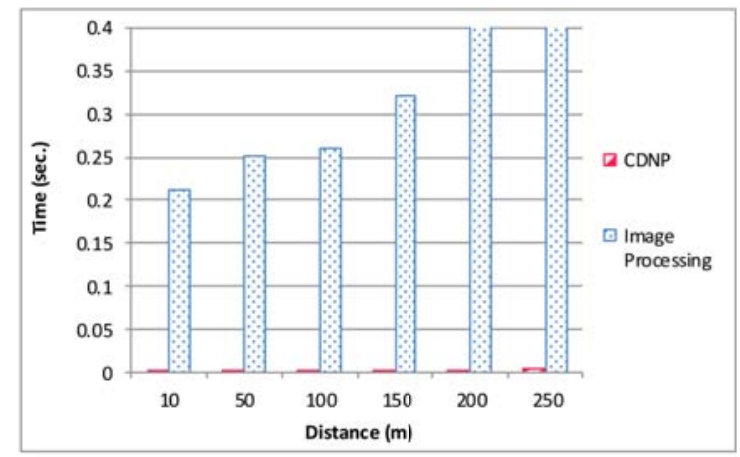

Figure 16. Identification time comparison.

- Identification Time Simulation Results

The Figure 16 shows the identification time comparison between CDNP and normal image processing based car light identification. Because the CDNP message is transmitted through the wireless technology, the transmission time is almost smaller than 0.002 seconds. Longer processing time is necessary for the image processing based identification to identify the car turn light correctly. The identifications of $200 \mathrm{~m}$ and $250 \mathrm{~m}$ are failed, so the processing times are infinity. Furthermore, although the image processing can still perform real-time identification, the image processing results may be incorrectly classified according to [15]. It will be dangerous if the result is incorrect. The CDNP identifies the driving decision according to the types and codes, so there is no incorrect identification.

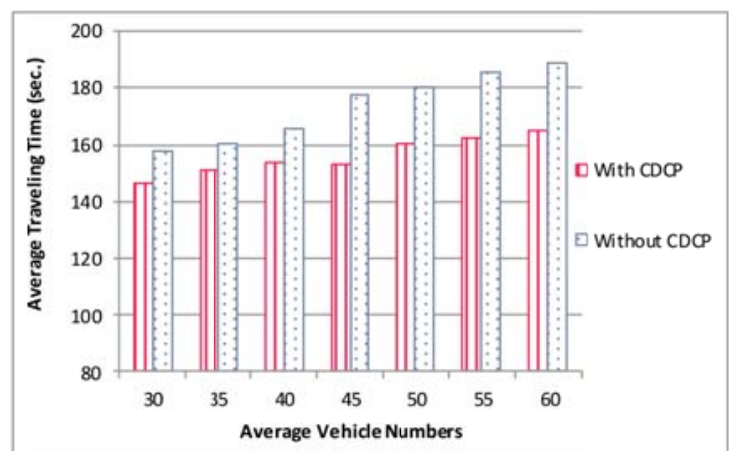

Figure 17. Traveling time of a vehicle traveling through the highway.

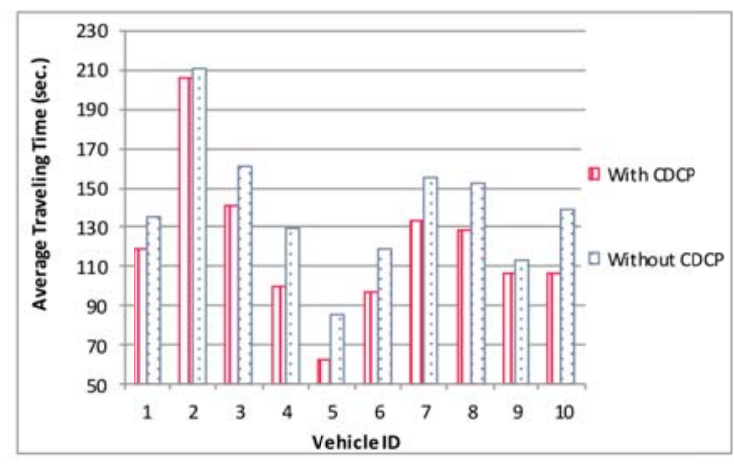

Figure 18. Traveling time of selected vehicles traveling from their start position to their end position in the urban scenario.

\section{- Vehicle Traveling Simulation Results}

One vehicle is selected to be observed in the highway scenario, and ten vehicles are selected to be observed in the urban scenario. The selected vehicles are configured with highest driving speed. The vehicle transfer overtake CDNP notification if it finds a slower vehicle in the front. The position and speed of other vehicles can be got from the beacon message (or called basic safety message) [20, 26-28]. The vehicles which received the notification will calculate the relative position of surrounding vehicles and check whether it is possible to change its driving lane according to the safe distance rule. If it is not possible, the danger reply will be sent. If it is possible, the simulator changes the driving lane using TraCI.

The results of this simulation are shown as Figure 17 and Figure 18. It is shown that the CDNP can decrease the average traveling time. Actually, the purpose of this simulation is to show the possible driving concept. The CDNP can be used as the function of the car horn. Moreover, it is better than the 
function of car horns, because the relative position of source vehicle can be easily determined according to the content of beacon information and CDNP packet. This simulation results can be considered as that the ambulance transported the injured. It notifies other vehicles make a way. The actually implementation of autonomous driving control rules depends on the car manufactures, and also must follow the traffic law of each country.

The reaction preparing time and identification time can be considered as the safety index. Longer reaction preparing time and shorter identification time can increase the processing time for the autonomous driving control system to make a better control of the autonomous driving.

\subsection{Discussion of Vehicle Moving Charac- teristic based on the CDNP}

In this section, the characteristics of autonomous vehicle movement related to the CDNP are described, including reaction time and traveling time.

Theorem: Let $v_{1}$ and $v_{2}$ be the speed of the vehicle 1 and vehicle 2 respectively, and the vehicle 1 runs after the vehicle 2. $T_{\text {range }}$ is the transmission range of VANET. For the driving decisions, e.g., overtaking, braking, turning driving direction, of vehicle 1 / vehicle 2 , the maximum reaction preparing time $\left(R P T_{m}\right)$ of vehicle $2 /$ vehicle 1 is given as

$$
R P T_{\text {max }}=T_{\text {range }} /\left|\left(\mathrm{v}_{1}-\mathrm{v}_{2}\right)\right|
$$

Proof: The transmission range of VANET technology is $T_{\text {range }}$ meters, so each vehicle can communicate with other vehicles when the distance is shorter than $T_{\text {range }}$ meters. Hence, the maximum reaction time is the time between the vehicles $1 /$ vehicle 2 enters the transmission range of the vehicle $2 /$ vehicle 1 , until the vehicle $1 /$ vehicle 2 wants to perform its driving decision. Because length equals speed multiplied by time, obviously, the reaction time $R P T_{m}$ is $T_{\text {range }} /\left(\left|v_{1}-v_{2}\right|\right)$.

\section{Conclusion}

The simulation tools, NS-3 and SUMO, are used to perform the simulations and evaluations of the proposed protocol. The programming languages used are $\mathrm{C}++$. The wireless transmission simula- tion, reaction preparing time simulation, identification time simulation, and vehicle travelling simulation are done in the simulation.

The simulation results show that the CDNP message can be transmitted within 0.002 seconds using VANET technology. It also shows that the CDNP increases the reaction preparing time in some situations. This can provide one autonomous vehicle with enough computation time to make a better driving decision. The maximum value of the reaction preparing time is 250 seconds if the relative speed between two vehicles is very close. It also decreases the driving decision identification time that is longer if sensor based method is used. The CDNP changes the traditional passive detection of the driving decision of other vehicles into proactive notification to other vehicles about its driving decision. Furthermore, the simulation results also show the CDNP can decrease the traveling time in special case. It can be considered as the scenario that the ambulance transported the injured. It notifies other vehicles make a way. It also means the autonomous driving is smoother and the carbon dioxide emissions could be reduced in some situation. From the theorem, it is shown that the maximum reaction preparing time is $R P T_{m}$.

The Intelligent vehicular ad hoc networks (InVANETs) and telematics technology, also known as ICT (Information and Communications Technology), are rapidly innovated to improve the development of ITS (Intelligent Transportation System). More and more vehicles are going to equip with invehicle telematics systems (or VANET wireless interface) to provide the communication capabilities to gather information from other vehicles (V2V) or internet (V2R). Furthermore, the autonomous driving and autonomous vehicle are important technologies for the future intelligent transportation system. To realize the autonomous driving in our daily life, the cooperation between vehicles is necessary. For the cooperation, the most important is information exchange. Unsafe driving decision, e.g. unsafe lane changes, unsafe overtake, or unsafe speed changes, will cause vehicle accidents; especially a vehicle suddenly performs those driving decisions. The CDNP provide the autonomous vehicles with the capability of understanding what detail driving decisions that other vehicles want to perform. This kind of idea is useful to improve the development 
of Intelligent Transportation Systems (ITS) and its services, and makes the driving environment safer in the future. The CDNP can also be integrated with the normal autonomous driving technologies at the same time to provide a safer driving environment for passengers. The CDNP can be a globally consistent standard to be abided by all car manufacturers and research centers. All car manufacturers and research centers can implement their autonomous driving technologies based on the CDNP. The CDNP is going to be implemented as a module at the network protocol stack in the Linux kernel to provide the basic protocol capability of the future development of autonomous vehicles in intelligent transportation system and its services in the future. This proposal is also going to develop an embedded system with VANET wireless interface and CDNP to do the real world wireless transmission test.

\section{References}

[1] Q. Li, L. Chen, M. Li, S. L. Shaw, A. Nuchter, A Sensor-Fusion Drivable-Region and Lane-Detection System for Autonomous Vehicle Navigation in Challenging Road Scenarios, IEEE Transactions on Vehicular Technology, 63, 2014, pp. 540-555.

[2] K. Chu, M. Lee, M. Sunwoo, Local Path Planning for Off-Road Autonomous Driving With Avoidance of Static Obstacles, IEEE Transactions on Intelligent Transportation Systems, 13, 2012, pp. 1599-1616.

[3] A. Broggi, M. Buzzoni, S. Debattisti, P. Grisleri, M.C. Laghi, P. Medici, P. Versari, Extensive Tests of Autonomous Driving Technologies, IEEE Transactions on Intelligent Transportation Systems, 14, 2013, pp. 1403-1415.

[4] J. Choi, J. Lee, D. kim, G. Soprani, P. Cerri, A. Broggi, $\mathrm{K}$ Yi, Environment-Detection-andMapping Algorithm for Autonomous Driving in Rural or Off-Road Environment, IEEE Transactions on Intelligent Transportation Systems, 13, 2012, pp. 974-982.

[5] J. Hardy, M. Campbell, Contingency Planning Over Probabilistic Obstacle Predictions for $\mathrm{Au}-$ tonomous Road Vehicles, IEEE Transactions on Robotics, 29, 2013, pp.913-929.

[6] Y. Toor, P. Muhlethaler, A. Laouiti, Vehicle Ad Hoc networks: applications and related technical issues, IEEE Communications Surveys \& Tutorials, 10, 2008, pp. 74-88.
[7] L. Guvenc, I. M. C. Uygan, K. Kahraman, R. Karaahmetoglu, I. Altay, M. Senturk, M. T. Emirler, A. E. H. Karci, B. A. Guvenc, E. Altug, M. C. Turan, O. S. Tas, E. Bozkurt, U. Ozguner, K. Redmill, A. Kurt, B. Efendioglu, Cooperative Adaptive Cruise Control Implementation of Team Mekar at the Grand Cooperative Driving Challenge, IEEE Transactions on Intelligent Transportation Systems, 13, 2012, pp.1062-1074.

[8] C. Lin, F. Dong, K. Hirota, Fuzzy Inference Based Vehicle to Vehicle Network Connectivity Model to Support Optimization Routing Protocol for Vehicular Ad-Hoc Network (VANET), Journal of Advanced Computational Intelligence and Intelligent Informatics, 18, 2014, pp. 9-12.

[9] X. Ma, J. Zhang, X. Yin, K.S. Trivedi, Design and Analysis of a Robust Broadcast Scheme for VANET Safety-Related Services, IEEE Transactions on Vehicular Technology, 61, 2012, pp. 4661.

[10] S. Mohammad, A. Rasheed, A. Qayyum, VANET Architectures and Protocol Stacks: A Survey, Communication Technologies for Vehicles, 6596, 2011, pp. 95-105.

[11] C.-Y. Yang, S.-C. Lo, Street Broadcast with Smart Relay for Emergency Messages in VANET, 2010 IEEE 24th International Conference on Advanced Information Networking and Applications Workshops (WAINA), 2010, pp. 323-328.

[12] J.-H. Lim, W. Kim, K. Naito, M. Gerla, Interplay between TVWS and DSRC: Optimal strategy for QoS of safety message dissemination in VANET, 2013 International Conference on Computing, Networking and Communications (ICNC), 2013, pp. 1156-1161.

[13] S. Kato, S. Tsugawa, K. Tokuda, T. Matsui, H. Fujii, Vehicle control algorithms for cooperative driving with automated vehicles and intervehicle communications, IEEE Transactions on Intelligent Transportation Systems, 3, 2002, pp. 155-161.

[14] L. Li, F.-Y. Wang, Cooperative Driving at Blind Crossings Using Intervehicle Communication, IEEE Transactions on Vehicular Technology, 55, 2006, pp. 1712-1724.

[15] M. Casares, A. Almagambetov, S. Velipasalar, A Robust Algorithm for the Detection of Vehicle Turn Signals and Brake Lights, 2012 IEEE Ninth International Conference on Advanced Video and Signal-Based Surveillance (AVSS), 2012, pp. 386391.

[16] Y. Li, Z.-X. Cai, J. Tang, Recognition algorithm for turn light of front vehicle, Journal of Central South University, 19, 2012, pp. 522-526. 
[17] NS-3, from http://www.nsnam.org/.

[18] M. Behrisch, L. Bieker, J. Erdmann, D. Krajzewicz, SUMO-Simulation of Urban MObility-an Overview, The Third International Conference on Advances in System Simulation, Barcelona, Spain, 2011, pp. 55-60.

[19] IEEE Standards Association, IEEE 802.11p-2010: Wireless LAN Medium Access Control (MAC) and Physical Layer (PHY) Specifications Amendment 6: Wireless Access in Vehicular Environments, 2010.

[20] DSRC Committee, Dedicated short range communications (DSRC) message set dictionary, SAE Standard J2735, SAE Internal, 2009.

[21] IEEE Standards Association, IEEE standard for wireless access in vehicular environments (WAVE) -multi-channel operation, 2010.

[22] Q. Yang, A. Lim, S. Li, J. Fang, P. Agrawal, ACAR: Adaptive Connectivity Aware Routing for Vehicular Ad Hoc Networks in City Scenarios. Mobile Networks and Applications, 15, 2010, pp. 36-60.

[23] W. Viriyasitavat, O. K. Tonguz, F. Bai, UV-CAST: an urban vehicular broadcast protocol, IEEE Communications Magazine, 49, 2011, pp. 116-124.

[24] M. Slavik, I. Mahgoub, Spatial Distribution and Channel Quality Adaptive Protocol for Multihop Wireless Broadcast Routing in VANET, IEEE Transactions on Mobile Computing, 12, 2013, pp. 722-734.

[25] ETSI EN 302665 V1.1.1 (2010-09). Intelligent Transport Systems (ITS), 2010.

[26] X. Yin, X. Ma, K. S. Trivedi, MAC and application level performance evaluation of beacon message dissemination in DSRC safety communication, Performance Evaluation, 71, 2014, pp. 1-24.
[27] J. B. Kenney, Dedicated Short-Range Communications (DSRC) Standards in the United States, Proceedings of the IEEE, 99, 2011, pp. 1162-1182.

[28] ETSI TC 102 637-2 V1.2.1 (2011-03) Intelligent Transport Systems (ITS); Vehicular Communications; Basic Set of Applications; Part 2: Specification of Cooperative Awareness Basic Service, 2011.

[29] L. Zhang, B. Jin, Dubhe: A Reliable and Low-Latency Data Dissemination Mechanism for VANETs, International Journal of Distributed Sensor Networks, doi:10.1155/2013/581821, 2013.

[30] H. Saleet, O. Basir, R. Langar, R. Boutaba, RegionBased Location-Service-Management Protocol for VANETs, IEEE Transactions on Vehicular Technology, 59, 2010, pp. 917-931.

[31] E. Lochin, G. Jourjon, S. Ardon, Design and validation of a reliable rate based transport protocol: The Chameleon protocol, Global Information Infrastructure Symposium (GIIS '09), 2009, pp. 1-8.

[32] A. Wegener, M. Piorkowski, M. Raya, H. Hellbrck, S. Fischer, J. P. Hubaux, TraCI: An Interface for Coupling Road Traf?c and Network Simulators, 11th Commun. and Networking Simulation Symposium (CNS'08), 2008, pp. 155-163.

[33] Openstreetmap, from http://www.openstreetmap.org/.

[34] Q. Luo, L. Xun, Z. Cao, Y. Huang, Simulation analysis and study on car-following safety distance model based on braking process of leading vehicle, 2011 9th World Congress on Intelligent Control and Automation (WCICA), 2011, pp. 740-743. 